\title{
Corrigendum: Differential translation efficiency of orthologous genes is involved in phenotypic divergence of yeast species
}

Orna Man \& Yitzhak Pilpel

Nat. Genet. 39, 415-421 (2007); published online 4 February 2007; corrected after print 28 March 2007

In Figure 2 of the original version of this paper, panels $\mathbf{c}$ and $\mathbf{d}$ were accidentally transposed, resulting in incorrect information in the legend. Figure 2 $\mathbf{c}$ shows genes of the tricarboxylic acid (TCA) cycle, and Figure 2d shows glycolysis genes. The error has been corrected in the HTML and PDF versions of the article.

Corrigendum: A common coding variant in CASP8 is associated with breast cancer risk

Angela Cox, Alison M Dunning, Montserrat Garcia-Closas, Sabapathy Balasubramanian, Malcolm W R Reed, Karen A Pooley, Serena Scollen, Caroline Baynes, Bruce A J Ponder, Stephen Chanock, Jolanta Lissowska, Louise Brinton, Beata Peplonska, Melissa C Southey, John L Hopper, Margaret R E McCredie, Graham G Giles, Olivia Fletcher, Nichola Johnson, Isabel dos Santos Silva, Lorna Gibson, Stig E Bojesen, Børge G Nordestgaard, Christen K Axelsson, Diana Torres, Ute Hamann, Christina Justenhoven, Hiltrud Brauch, Jenny Chang-Claude, Silke Kropp, Angela Risch, Shan Wang-Gohrke, Peter Schürmann, Natalia Bogdanova, Thilo Dörk, Rainer Fagerholm, Kirsimari Aaltonen, Carl Blomqvist, Heli Nevanlinna, Sheila Seal, Anthony Renwick, Michael R Stratton, Nazneen Rahman, Suleeporn Sangrajrang, David Hughes, Fabrice Odefrey, Paul Brennan, Amanda B Spurdle, Georgia Chenevix-Trench, The Kathleen Cunningham Foundation Consortium for Research into Familial Breast Cancer, Jonathan Beesley, Arto Mannermaa, Jaana Hartikainen, Vesa Kataja, Veli-Matti Kosma, Fergus J Couch, Janet E Olson, Ellen L Goode, Annegien Broeks, Marjanka K Schmidt, Frans B L Hogervorst, Laura J Van’t Veer, Daehee Kang, Keun-Young Yoo, Dong-Young Noh, Sei-Hyun Ahn, Sara Wedrén, Per Hall, Yen-Ling Low, Jianjun Liu, Roger L Milne, Gloria Ribas, Anna Gonzalez-Neira, Javier Benitez, Alice J Sigurdson, Denise L Stredrick, Bruce H Alexander, Jeffery P Struewing, Paul D P Pharoah \& Douglas F Easton, on behalf of the Breast Cancer Association Consortium Nat. Genet. 39, 352-358 (2007); published online 11 February 2007; corrected 10 April 2007

In the version of this article initially published, there was an error that affected the calculations of the odds ratios, confidence intervals, betweenstudy heterogeneity, trend test and test for association for SNP ICAM5 V301I in Table 1 (ICAM5 V301I); genotype counts in Supplementary Table 2 (ICAM5; ICR_FBCS and Kuopio studies) and minor allele frequencies, trend test and odds ratios for heterozygotes and rare homozygotes in Supplementary Table 3 (ICAM5; ICR_FBCS and Kuopio studies). The corrected rows from each table are reproduced below.

The errors in Table 1 have been corrected in the PDF version of the article. The errors in supplementary information have been corrected online.

Table 1 Summary odds ratios and $95 \%$ confidence intervals for nine polymorphisms and breast cancer risk

\begin{tabular}{lllllll}
\hline SNP & $\begin{array}{l}\text { Between-study hetero- } \\
\text { geneity }\end{array}$ & Test for association & Trend test & Analysis model & Heterozygote OR (95\% c.i.) & $\begin{array}{l}\text { Rare homozygote } \\
\text { OR (95\% c.i.) }\end{array}$ \\
\hline ICAM5 V301I & 0.57 & 0.54 & 0.98 & Fixed effects & $1.02(0.97,1.07)$ & $0.99(0.93,1.05)$ \\
rs1056538 & & & & Random effects & $1.02(0.97,1.07)$ & $0.99(0.93,1.05)$
\end{tabular}

Supplementary Table 2 Genotype counts among cases and controls by study

\begin{tabular}{|c|c|c|c|c|c|c|c|c|c|c|}
\hline \multirow[b]{2}{*}{ Gene } & \multirow[b]{2}{*}{ SNP } & \multirow[b]{2}{*}{ Study } & \multicolumn{4}{|c|}{ Controls } & \multicolumn{4}{|c|}{ Cases } \\
\hline & & & $\overline{\mathrm{AA}}$ & $\mathrm{Aa}$ & aa & Total & AA & $\mathrm{Aa}$ & aa & Total \\
\hline ICAM5 & rs1056538 & ICR_FBCS & 207 & 243 & 71 & 521 & 212 & 239 & 68 & 519 \\
\hline
\end{tabular}

Supplementary Table 3 Association between nine polymorphisms and breast cancer risk by study

\begin{tabular}{|c|c|c|c|c|c|c|c|c|c|c|}
\hline \multirow{3}{*}{$\begin{array}{l}\text { Gene } \\
\text { ICAM5 }\end{array}$} & \multirow{3}{*}{$\begin{array}{l}\text { SNP } \\
\text { rs1056538 }\end{array}$} & \multirow{3}{*}{$\begin{array}{l}\text { Study } \\
\text { ICR_FBCS }\end{array}$} & \multirow{3}{*}{$\begin{array}{l}\text { MAF } \\
0.369\end{array}$} & \multirow{3}{*}{$\begin{array}{l}\text { Trend test } \\
0.698\end{array}$} & \multicolumn{3}{|c|}{ Heterozygotes } & \multicolumn{3}{|c|}{ Rare homozygotes } \\
\hline & & & & & \multirow{2}{*}{$\begin{array}{c}\text { OR } \\
0.960\end{array}$} & \multicolumn{2}{|c|}{ 95\% c.i. } & \multirow{2}{*}{$\begin{array}{c}\text { OR } \\
0.935\end{array}$} & \multicolumn{2}{|c|}{ 95\% c.i. } \\
\hline & & & & & & 0.739 & 1.248 & & 0.637 & 1.373 \\
\hline & & Kuopio & 0.348 & 0.658 & 0.909 & 0.687 & 1.203 & 0.962 & 0.612 & 1.513 \\
\hline
\end{tabular}

